\title{
The use of local anaesthesia for intrauterine device insertion by health professionals in the UK
}

\author{
Hannat Akintomide, ${ }^{1}$ Robert D E Sewell, ${ }^{2}$ Judith M Stephenson ${ }^{3}$
}

\begin{abstract}
${ }^{1}$ Specialty Doctor, Camden Provider Services - Sexual \& Reproductive Health, Margaret Pyke Centre, London, UK ${ }^{2}$ Reader in Pharmacology, Welsh School of Pharmacy, Cardiff University, Cardiff, UK ${ }^{3}$ Margaret Pyke Professor of Sexual \& Reproductive Health, Institute for Women's Health, University College London, London, UK
\end{abstract}

\section{Correspondence to}

Dr Hannat Akintomide, Camden Provider Services - Sexual \& Reproductive Health, Margaret Pyke Centre, 73 Charlotte Street, London W1T 4PL, UK;

h.akintomide@nhs.net

Received 11 September 2012 Accepted 18 December 2012

Published Online First

5 February 2013
To cite: Akintomide $H$, Sewell RDE, Stephenson JM. J Fam Plann Reprod Health Care 2013;39:276-280.

\section{ABSTRACT}

Background Pain associated with the insertion of an intrauterine device (IUD) is a known barrier to intrauterine contraception use in the UK. It is good practice for health professionals to discuss pain relief and use with women prior to the insertion of an IUD.

Objectives This study aimed to determine the prevalence of and reasons for and against the use of local anaesthesia (LA) for IUD insertion. Methods A survey was undertaken using paper questionnaires to determine LA use for IUD insertion by UK health professionals.

Results Overall, approximately one quarter $(n=129)$ of all respondents use LA routinely, one quarter hardly ever or never use LA, while the remaining half use it sometimes. Use of LA was more prevalent among health professionals who worked in integrated sexual and reproductive health and contraception-only services, compared to general practice. UK health professionals who hardly ever or never used LA for the insertion of IUDs were more likely to be working in general practice.

Conclusions The results of this survey suggest that more UK health professionals need to routinely discuss pain relief and offer this to their patients prior to IUD insertion as part of the care pathway for patients who choose to use intrauterine contraception.

\section{BACKGROUND}

Increasing uptake of long-acting reversible contraception (LARC) is an important component of strategies to reduce the rates of unintended pregnancy and abortion in the UK. Intrauterine contraception is a LARC method that is more cost effective, has higher user continuation rates and offers other benefits to women compared to other LARC methods. ${ }^{1}$ The procedure for the insertion of an intrauterine device (IUD) is associated with pain and/or discomfort in some women,

\section{Key message points}

- It is good practice for health professionals to discuss pain relief and its use with women prior to the insertion of an intrauterine device.

- Most UK health professionals do not routinely use local anaesthesia (LA) for the insertion of intrauterine contraception.

- UK health professionals should routinely discuss pain relief and always consider use of LA for intrauterine contraception insertion in women during contraceptive consultations.

which is a known barrier to intrauterine contraception use. Health professionals differ in their opinions on women's perceptions of pain or discomfort, as well as about the use of local anaesthesia (LA) for IUD insertions. ${ }^{2}$ There is also no form or type of analgesic or local anaesthetic licensed for IUD insertion that is proven to be of value in reducing IUD insertion pain. ${ }^{3}$ These factors conceivably influence health professionals' decisions to use currently available LA routinely for IUD insertions. There are, however, few available data on the opinions and practice of health professionals in the UK as regards this issue. Consequently, a survey was performed in order to determine first the prevalence of routine LA use for IUD insertion by UK health professionals, and second, the reasons for and against the use of LA.

\section{METHODS}

A questionnaire survey was used to obtain opinions and information about the practice of UK health professionals who insert IUDs. The questionnaire was developed following a review of the scientific literature, consideration of the UK's Faculty of Sexual \& Reproductive 
Healthcare (FSRH) recommendations ${ }^{4}$ and interviews with experienced health professionals who currently insert IUDs. The questionnaire was validated and piloted in a sample $(n=10)$ of experienced health professionals working in a contraceptive service. It was anonymous and self-administered. The seven item semi-structured questionnaire was printed on a single sheet, participant information being requested on the reverse side. The questionnaire was designed to take about 1 minute to complete and posed questions about the use of LA for IUD insertions, the type of LA used, reasons for infrequent or non-use of LA, and the experience and current practice of the health professional concerned. Participants could select or supply more than one reason for and/or against their use of LA for IUD insertions.

The survey was carried out at a Current Choices Conference held in London, UK in November 2011. This conference is an annual academic event organised by the FSRH. The 2-day conference is open to all doctors, nurses and allied health professionals who work or have an interest in sexual and reproductive health (SRH). Delegates attended the conference from all over the UK, however not all of them performed IUD insertions.

\section{Data analysis}

Questionnaire responses were collected and collated using a Google Docs ${ }^{\mathrm{TM}}$ Spreadsheet. Values for $p$ were calculated using Fisher Exact tests and contingency tables, with frequency of use of LA for IUD insertion as outcomes.

\section{Ethical approval}

This survey of health professionals who insert IUDs was exempt from review by an ethics committee.

\section{RESULTS}

A total of 313 delegates attended the conference, half of whom were extrapolated to be health professionals who inserted IUDs from current data held by the FSRH. A total of 129 questionnaires were returned, although 19 of these were not fully completed, giving a response rate of $82 \%(129 / 157)$. Most respondents were manifestly experienced: $86 \%$ had been carrying out IUD insertions for over 5 years and $67 \%$ had performed up to 50 or more IUD insertions in the previous 12 months. About half (44\%) of the respondents worked mainly in an integrated SRH (both genitourinary medicine and contraception) service, while almost equal proportions of respondents worked mainly in general practice $(29 \%)$ or a contraception-only service (24\%). Only four respondents worked mainly in a hospital setting (Table 1).

Seventy-five percent of the respondents used LA for IUD insertions ( $28 \%$ always, $47 \%$ sometimes), while the remainder did not use or hardly ever used LA. The health professionals who always used LA, or only

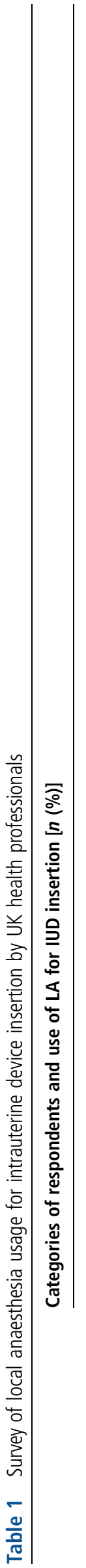


sometimes used LA for IUD insertions $(n=96)$, worked mainly in integrated SRH services (48\%, $n=46)$, in comparison to general practice $(25 \%$, $n=24)$, contraception-only services $(24 \%, n=23)$ or a hospital setting $(3 \%, n=3)$. General practice had the highest proportion $(41 \%, n=32)$ of health professionals who hardly ever used or did not use LA, compared to those working in integrated SRH (31\%) or contraception-only services (25\%). Topical gel LA was the commonest type of LA used by UK health professionals, irrespective of whether they always used LA for IUD insertions, only used LA sometimes or hardly ever used LA for IUD insertions (Table 1).

Of the total number $(n=87)$ of health professionals who ever used (both always and sometimes) topical gel LA for IUD insertions, $60 \%(n=52)$ usually used topical gel LA alone while the remainder $(n=37)$ used it in conjunction with injectable LA. Seventy-eight of these health professionals allowed less than 5 minutes, six allowed 6-10 minutes, while three health professionals allowed more than 10 minutes for the topical gel LA to act during the IUD insertion procedure.

Seventy-five respondents gave their reasons for and against the use of LA for IUD insertions. The commonest reasons for not using LA were that IUD insertion did not require LA (despite an acceptance by the same respondents that the IUD insertion procedure was associated with pain) and that using LA may itself cause pain and prolong the procedure. The least common reasons cited for LA use included if the patient was nulliparous, if the patient was anxious, and if the patient requested LA (Table 2).

\section{DISCUSSION}

This survey found that less than one-third of health professionals always used LA for IUD insertions. The main reason given for non-use of LA is the belief that the pain associated with IUD insertion does not warrant this form of pain relief. Only a few health professionals cited non-availability of LA or LA facilities or costs as reasons for not always using LA for IUD insertions.

Health professionals who always used LA for IUD insertion worked mainly in integrated SRH and contraception services, whereas health professionals who did not use or rarely used LA were more likely to be working in general practice. When health professionals did use LA for IUD insertion, this was more likely to be topical gel LA only, which was allowed no more than 5 minutes to take effect.

This survey examined the use of LA for IUD insertion by health professionals. It was carried out at an FSRH conference in the UK attended by more than 300 delegates. This setting maximised the inclusion of as many IUD-inserting health professionals as possible at a single event. The short, semi-structured anonymous questionnaire encouraged health professionals to participate and answer all the questions. The survey was confidential and completed questionnaires could be returned at any time during the 2-day conference.

Due to the 'opt in' design of the survey, the exact response rate cannot be calculated. However, based on current data that $53 \%$ of health professionals registered with the FSRH also hold Letters of Competence to insert IUDs, it is reasonable to extrapolate that up to half of the conference's attendees insert IUDs. This

Table 2 Reasons given by UK health professionals against and for the use of local anaesthesia for intrauterine device insertion

\begin{tabular}{|c|c|}
\hline Reasons given & $n(\%)$ \\
\hline \multicolumn{2}{|l|}{ Reasons against the use of LA } \\
\hline In my experience, IUD insertion could be painful but the pain only lasts a short time (LA not required) & $27(19)$ \\
\hline In my experience, IUD insertion could be painful but does not require LA & $16(11)$ \\
\hline In my experience, LA administration takes time, which prolongs the procedure itself & $16(11)$ \\
\hline In my experience, more pain is caused by LA administration than the IUD insertion procedure & $16(11)$ \\
\hline I have not been trained to use LA for IUD insertions & $9(6)$ \\
\hline LA is not available in the centre where I work & $9(6)$ \\
\hline There is no evidence that suggests LA is effective for pain with IUD insertion & $7(5)$ \\
\hline In my experience, LA is not effective & $5(4)$ \\
\hline In my experience, IUD insertion is not painful & $5(4)$ \\
\hline There are no facilities for LA administration in the centre where I work & $4(3)$ \\
\hline LA is expensive for the centre where I work & $3(2)$ \\
\hline Current evidence suggests that available LA is not effective for pain with IUD insertion & $3(2)$ \\
\hline \multicolumn{2}{|l|}{ Reasons for the use of LA } \\
\hline I use LA if the IUD insertion is painful for the patient & $5(4)$ \\
\hline I use LA when IUD insertion requires dilatation & $4(3)$ \\
\hline I use LA if the patient is anxious & $4(3)$ \\
\hline I use LA if the patient requests it & $4(3)$ \\
\hline I use LA if the patient is nulliparous & $3(2)$ \\
\hline
\end{tabular}

IUD, intrauterine device; LA, local anaesthesia. 
would infer that the 129 completed questionnaires reflected a response rate of $82 \%(129 / 157)$.

The sample surveyed during the FSRH conference may not be representative of health professionals who insert IUDs in the UK or the prevalence of LA use for IUD insertion across the UK's health services. In addition, the FSRH conference was more likely to have been attended by practitioners who worked in SRH services rather than other settings. For example, only four of the survey participants worked mainly in a hospital service; $29 \%$ of respondents worked mainly in general practice, whereas up to $60 \%$ of health professionals registered with the FSRH are believed to be general practitioners (GPs). Nevertheless, current estimates and prescribing data suggest that although GPs provide $75 \%$ and community clinics (SRH services) provide $25 \%$ of contraception services to women, LARC provision (including IUD insertions) by community clinics is greater than that provided by GPs. ${ }^{15}{ }^{6}$ These data therefore make the proportion of GP respondents in this survey significant.

The prevalence of LA use for IUD insertion by UK health professionals demonstrated by this survey is similar to the findings of Tolcher $^{2}$ who determined LA use, amongst other practices surrounding IUD insertions, across a single SRH service. Tolcher's study found that most health professionals used topical gel LA for IUD insertion but only sometimes (72\%), in comparison to those who always $(16.7 \%)$ or rarely/ never (11\%) did. ${ }^{2}$ However, Tolcher did not determine the time period that health professionals allowed for the topical gel LA to take effect.

The fear of pain during the insertion procedure is a known deterrent for women choosing to use an IUD. Despite minimal evidence that LA or analgesia are wholly effective in reducing or eliminating pain associated with IUD insertion, it remains good practice to discuss, offer and use available LA for a procedure that may cause pain or discomfort. ${ }^{4}$ Pain control should be an objective of all health professionals in their routine practice when providing care.

The attitudes and arguments of health professionals against always offering and using LA have included LA being unnecessary, unwanted by patients, and over-medicalisation of the IUD insertion procedure. ${ }^{78}$ These reasons are similar to those mostly cited by health professionals in our survey against routine LA use for IUD insertion. There is no evidence for these arguments in the scientific literature, and indeed they have been refuted. ${ }^{9-11}$ In contrast, there are reports that most patients are satisfied with the use of LA for IUD insertion, and are happy to undergo a repeat procedure involving LA in the future. ${ }^{12}{ }^{13}$ Moreover, other health professionals have reported LA use to be beneficial for IUD insertion. ${ }^{2} 14$

Time, training and efficacy were other reasons cited by health professionals for not routinely using LA for IUD insertions. It is possible that more health professionals used topical gel LA because they were not trained in the use of injectable LA which, unlike topical gel LA, has an almost immediate effect after administration. Though the use of topical gel LA was more prevalent in the current survey, its application was suboptimal since a contact time of less than 5 minutes was frequently allowed for the gel to take effect. For gynaecological procedures such as IUD insertion it is advisable to allow 5 minutes for Instillagel ${ }^{\circledR}$ (the only currently available lignocaine gel anaesthetic in the UK) to take effect. ${ }^{15-17}$ Where the use of Instillagel has been found to reduce associated pain with gynaecological procedures, including difficult IUD insertions and removals, 10-15 minutes are typically allowed for it to take effect. ${ }^{18}$ Health professionals who use topical gel LA should therefore allow a contact time of $5+$ minutes in order to optimise efficacy for IUD insertion pain. Our findings suggest that there may be a need for health professionals to learn techniques for topical gel LA administration in addition to injectable LA administration for IUD insertion.

\section{CONCLUSIONS}

This survey suggests that the effective use of currently available LA for IUD insertion is not substantial and could be increased amongst UK health professionals. The discussion, offer or provision of pain relief during IUD insertion is good practice but is usually at the discretion of the health professional performing the IUD insertion procedure or the service where the IUD insertion is being done. We recommend educating UK health professionals about LA use and acceptance as good practice, the provision of suitable equipment, and minimising the extra costs and/or time associated with LA administration to encourage more UK health professionals to discuss LA with their patients and use LA for IUD insertions.

Competing interests None.

Provenance and peer review Not commissioned; externally peer reviewed.

\section{REFERENCES}

1 National Intstitute for Health and Clinical Excellence (NICE). Long-acting Reversible Contraception. 2005. http://www.nice. org.uk/CG30 [accessed 18 February 2012].

2 Tolcher R. Intrauterine techniques: contentious or consensus opinion? J Fam Plann Reprod Health Care 2003;29:21-24.

3 Allen RH, Bartz D, Grimes DA, et al. Interventions for pain with intrauterine device insertion. Cochrane Database Syst Rev 2009;3:CD007373.

4 Faculty of Sexual \& Reproductive Healthcare Clinical Effectiveness Unit. Intrauterine Contraception. 2007. http:// www.fsrh.org/pdfs/CEUGuidanceIntrauterine ContraceptionNov07.pdf [accessed 18 February 2012].

5 Department of Health. Findings of the Baseline Review of Contraceptive Services. 2007. http://www.dh.gov.uk/en/ Publicationsandstatistics/Publications/PublicationsPolicyAnd Guidance/DH_074727 [accessed 18 February 2012]. 
6 Information Services Division Scotland Final Population Based KCI Report - LARC: 2009/2010 Data. 2010. http://www. isdscotland.org/Health-Topics/Sexual-Health/ Key-Clinical-Indicators/Final\%20KCI\%20LARC\%2020010\% 2027092010\%20(2).pdf [accessed 18 February 2012].

7 Jones K. Use of injectable local anaesthesia for IUD/IUS fitting. J Fam Plann Reprod Health Care 2011;37:253.

8 Devonald B. Injectable local anaesthetic for IUD/IUS fittings. J Fam Plann Reprod Health Care 2011;37:119.

9 Hutt S. Injectable local anaesthetic for IUD/IUS fittings: author's response. J Fam Plann Reprod Health Care 2011;37:188-189.

10 Hutt S. Option of local anaesthetic for IUD fittings: author's response. J Fam Plann Reprod Health Care 2011;37:190.

11 Hutt S. A fitting approach to coils. Br J Sex Med 2004;28:14-15.

12 Ronghe R, Groom T. IUD client satisfaction survey (Poster). Faculty of Sexual \& Reproductive Healthcare Annual Scientific Conference, Arena and Convention Centre, Liverpool, UK 12-13 May 2011.
13 Gebbie AE. Difficult IUD insertions. J Fam Plann Reprod Health Care 2008;34:134.

14 Ronghe R, Smart H, Groom T. Audit of use of local anaesthesia during intrauterine device insertion (Poster). Eur J Contraception Reprod Health Care 2010;15: 60-201.

15 CliniMed Ltd. Instillagel - Anaesthetic Antiseptic Lubricant, 'How the gel is used' (Company website). http://www.clinimed. co.uk/Urology-Continence-Care/Products/Instillagel.aspx [accessed 18 February 2012].

16 Navani M. Difficult IUD insertions. J Fam Plann Reprod Health Care 2008;34:135.

17 Draper IB. Difficult IUD insertions. J Fam Plann Reprod Health Care 2008;34:135.

18 Pillai M, Shefras J. Experience with Instillagel ${ }^{\circledR}$ for hysterosonography and analgesia in a complex contraception clinic: a QIPP initiative. J Fam Plann Reprod Health Care 2012;38:110-116. 